\title{
NMNAT3 improves mitochondrial function and enhances BMSCs anti-oxidative stress through the NAD+-Sirt3 pathway
}

\section{Tao Wang (D1911095370@qq.com )}

Guizhou Medical University https://orcid.org/0000-0002-4918-3915

\section{Wuxun Peng}

Guizhou Medical University

\section{Fei Zhang}

Guizhou Medical University

\section{Lei Wang}

Guiyang Maternity and Child Health Hospital

Jian Zhang

Guizhou Medical University

\section{Wentao Dong}

Guizhou Medical University

Chuan Ye

Guizhou Medical University

Xiaobin Tian

Guizhou Medical University

Yanlin Li

Kunming Medical University First Affilliated Hospital

\section{Yuekun Gong}

Kunming Medical University First Affilliated Hospital

\section{Research}

Keywords: oxidative stress, nicotinamide adenine dinucleotide, nicotinamide mononucleotide adenylyl transferase 3 , mitochondrial function, bone marrow mesenchymal stem cells

Posted Date: March 13th, 2020

DOI: https://doi.org/10.21203/rs.3.rs-17046/v1

License: (c) (1) This work is licensed under a Creative Commons Attribution 4.0 International License. Read Full License 


\section{Abstract}

Background To investigate the effects of NMNAT3 on mitochondrial function and anti-oxidative stress in rabbit BMSCs and its underlying mechanisms.

Methods Stable strains of NMNAT3 overexpressing rabbit BMSCs were obtained by lentivirus transfection; the Oxidative stress model in rabbit BMSCs was imitated by treating with $\mathrm{H} 2 \mathrm{O} 2$; Observe the changes in mitochondrial ultrastructure and mitochondrial function-related indicators (mitochondrial membrane potential, ATP and mitochondrial protein PGC-1a, NRF1 synthesis), to study the effect of NMNAT3 on improving mitochondrial function under oxidative stress; detect ROS and lipids Peroxidation products (MDA content), antioxidant enzymes (CAT and GPx) activity, SA- $\beta-G a l$ activity and apoptosis in rabbit BMSCs, to study the changes of the anti-oxidative stress ability of BMSCs modified by NMNAT3;Then, the selective Sirt3 inhibitor (3-TYP) was used to analyze the mechanism of NMNAT3 improve mitochondrial function and antioxidant stress ability of BMSCs by regulating mitochondrial NAD + levels, and whether it affects the acetylation levels of mitochondrial target proteins (Idh2 and FOXO3a) and the expression and activation of effect proteins (GSH, ATP synthase and Mn-SOD) through Sirt3 pathway.

Results Overexpression of NMNAT3 can improve the mitochondrial ultrastructural damage of rabbit BMSCs under oxidative stress; increase the mitochondrial membrane potential, increase the synthesis of ATP and mitochondrial protein PGC-1a and NRF1, significantly improve the function of mitochondria.It also can significantly enhance the antioxidant stress ability of rabbit BMSCs by reducing ROS level and MDA content, increasing the activities of antioxidant enzymes CAT and GPX, and decreasing cell senescence and apoptosis under stress.Rabbit BMSCs overexpressed by NMNAT3 significantly increased the activity of Sirt3, significantly decreased the acetylation levels of Idh2 and FOXO3a under oxidative stress, and increased the level of GSH and the activities of ATP synthase and Mn-SOD.However, the addition of 3-TYP basically blocked the protective effect of NMNAT3, indicating that Sirt3 is an important pathway for NMNAT3 to regulate mitochondrial function and antioxidant stress in BMSCs under oxidative stress.

Conclusion NMNAT3 can effectively improve the mitochondrial function of rabbit BMSCs under oxidative stress through NAD + -Sirt3 pathway, and play a role in enhancing its antioxidant stress injury.

\section{Background}

The survival of stem cell transplantation is the key problem to be solved in current research and clinical treatment ${ }^{\otimes 1-3 \rrbracket}$. Studies have shown that oxidative stress damage is often caused by the microenvironment such as ischemia, hypoxia and inflammation in the transplantation area, which seriously affects the mitochondrial function of stem cells and limits the proliferation, differentiation and survival of implanted stem cells. it accelerates the aging and apoptosis of transplanted stem cells, which

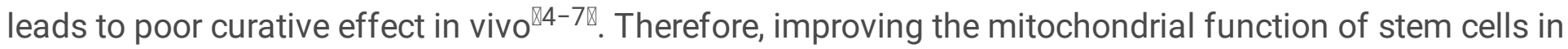


the transplantation area and enhancing its anti-stress ability may be an effective way to improve the survival rate and curative effect of stem cell transplantation ${ }^{88-11 \mathbb{~}}$.

In recent years, research on the molecular mechanism of mitochondrial disorders have shown that nicotinamide adenine dinucleotide $\left(\mathrm{NAD}^{+}\right)$is a key regulator in mitochondria, and the level of $\mathrm{NAD}^{+}$ directly controls important life processes such as cell rhythm, senescence, resistance and cytoprotection ${ }^{812-14 \rrbracket}$, which is mainly related to various enzymes dependent on $\mathrm{NAD}^{+}$. For example, involved in stress and aging-related protein deacetylation reactions, polyadenylate diphosphate ribose reactions that play important roles in DNA repair, and adenosine diphosphate ribose cyclization reactions that regulate

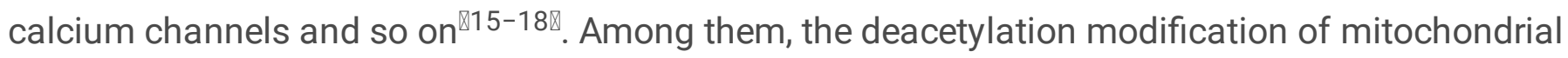
proteins plays an important role in regulating the functional stability of mitochondria and the adaptation to biological and chemical stressors ${ }^{\$ 19,20 \rrbracket}$, which is mainly mediated by the silent mating type information regulation 2 homolog 3 (Sirt3) of the sirtuins gene family ${ }^{821-24 区}$. The deacetylase activity of Sirt3 depends on $\mathrm{NAD}^{+}$activation. When the content of $\mathrm{NAD}^{+}$in mitochondria is depleted, it can lead to the decrease of Sirt3 activity, which directly affects the stability of energy metabolism and the balance of

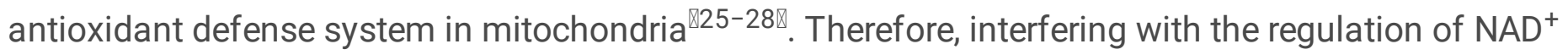
homeostasis in mitochondria and then improving the activity of Sirt3 is an important means to improve

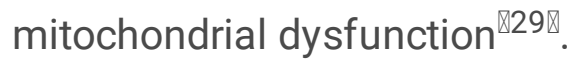

Among many enzymes involved in the regulation of $\mathrm{NAD}^{+}$homeostasis, nicotinamide mononucleotide adenylyl transferase 3 (NMNAT3) has been found to be essential for the formation of $N A D^{+}$in

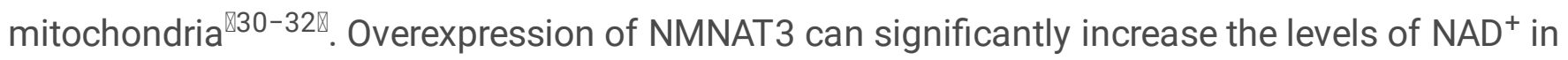
mitochondria of aged mouse tail-tip fibroblasts and human umbilical cord blood mesenchymal stem cells, restore Sirt3 activity, and delay cell aging and apoptosis ${ }^{\circledR 3}{ }^{\circledR}$. Similarly, Gulshan et al. have further demonstrated that NMNAT3 can be used as a target enzyme to increase the level of mitochondrial NAD ${ }^{+}$ in NMNAT3 transgenic mice, which is important to improve mitochondrial biosynthesis and energy metabolism, reduce the level of reactive oxygen species (ROS) and restore mitochondrial function ${ }^{\text {\34 }}$. However, at present, the effect of NMNAT3 on the mitochondrial function and anti-stress ability of stem cells in the transplantation area has not been studied, and it is unclear whether it will help improve the survival rate and efficacy of stem cell transplantation.

In this study, we overexpressed the NMNAT3 gene in bone marrow mesenchymal stem cells (BMSCs) in vitro. We simulated the microenvironment of oxidative stress in the transplantation area by $\mathrm{H}_{2} \mathrm{O}_{2}$ to evaluate the effects of NMNAT3 on the mitochondrial function and antioxidant stress ability of BMSCs

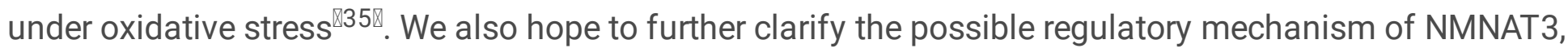
provide a new method to solve the problem of survival of stem cell transplantation, promote the clinical transformation of stem cells, and open up more new ideas for treatment.

\section{Materials And Methods}




\section{Animals}

all the experimental animals used in this study were provided by the Animal Experimental Center of Guizhou Medical University: A total of 20 young New Zealand white rabbits $(2.0 \pm 0.5 \mathrm{~kg})$. All animals are approved by the Experimental Animal Bioethics Committee of Guizhou Medical University (Grant No.1900590), and all procedures are carried out in strict accordance with the guidelines for the Care and use of Experimental Animals issued by the National Institutes of Health (NIH publication No. 85-23, 1996 revised).

\section{Cell culture}

The rabbits were fixed on the operating table of small animals in the supine position, and routinely disinfected and laid towels. The distal femur and proximal tibia of rabbits were anesthetized with $2 \%$ lidocaine hydrochloride (ShandongHualuPharmaceuticalCo.,Ltd.,China), and then punctured. The bone marrow solution was extracted from $4-5 \mathrm{~mL}$, and then the cells were separated by density gradient centrifugation ${ }^{\square 36,37 \square}$. The complete L-DMEM (Gibco,USA) medium containing 10\%FBS (Gibco,USA) and $1 \%$ double antibody (Hyclon,USA) was inoculated in the $25 \mathrm{~cm}^{2}$ culture bottle (Corning,USA) and cultured at $37{ }^{\circ} \mathrm{C}$ in the $5 \% \mathrm{CO}_{2}$ incubator (Thermo,USA). After that, the complete medium was changed every 3 days, and when the confluence of the cells at the bottom of the culture bottle reached more than $90 \%$, the BMSCs began to be digested and passaged at 1:3.

\section{Mitochondrial extraction}

The treated BMSCs was washed with PBS, the cells were digested by trypsin, and the cells were collected and counted. $5 \times 10^{7}$ cells were extracted and added to $1.0 \mathrm{~mL}$ ice-precooled PBS resuspended cells. The cell suspension was transferred into a small volume glass homogenizer and grinded in an ice bath at 0 ${ }^{\circ} \mathrm{C}$ for 30 times to obtain cell homogenate. Isolation and extraction of mitochondria according to the steps of the cell mitochondria separation kit (Beytime,China), and the mitochondrial precipitation was resuscitated with 50-100 $\mu \mathrm{L}$ Store Buffer or suitable reaction buffer, and then used immediately or stored at $-70^{\circ} \mathrm{C}$.

\section{BMSCs surface antigen identification}

The third generation of rabbit BMSCs, was digested and centrifuged to adjust the cell concentration to make $2.0 \times 10^{7}$ cells $/ \mathrm{mL}$ single cell suspension. $50 \mu \mathrm{L}$ cell suspension was taken into the flow tube (Corning,USA) and added with Anti-CD29/AF647, Anti-CD90/PE-Cy ${ }^{\mathrm{TM}} 7$, Anti-CD106/PE, Anti-CD45/FITC and Anti-CD11b/V450 (BD,USA), respectively. Buffer (Hyclon,USA) was used as negative control, and the 
supernatant was centrifuged to remove the supernatant after incubation at room temperature for $30 \mathrm{~min}$, $1000 \mathrm{rpm} / \mathrm{min}$ and $5 \mathrm{~min}$. Each tube was washed with buffer twice, and then 500uLbuffer resuspension cells were added to each tube and detected by flow cytometry (Beckman,USA).

\section{BMSCs multidirectional differentiation induction}

The third generation of rabbit BMSCs, with good growth was selected and the cell concentration was adjusted to $2.0 \times 10^{4}$ cells $/ \mathrm{cm}^{2}$, according to osteogenic, adipogenic and chondrogenic induction differentiation kit (CyagenBiosciencesSuzhoulnc.,China). The cells were inoculated on a 6-well plate with $2 \mathrm{ml}$ per well. When the cell convergence degree reached $60 \%, 100 \%$ and $60 \%$ respectively, the experimental group changed the induced differentiation medium respectively, while the control group continued to use complete L-DMEM medium. The operation is carried out according to the instructions of each induction kit. After the induction is completed, the culture medium is absorbed, washed twice with PBS (Hyclon,USA), 4\% paraformaldehyde (BeijingLeageneBiotech.Co.,Ltd.,China) of $1 \mathrm{~mL}$ is added to each well, fixed at room temperature for 30 minutes, and each well is washed twice with PBS buffer, according to the staining instructions of each kit, Alizarin red staining solution, oil red $\mathrm{O}$ staining solution and alisin blue staining solution were used for staining (CyagenBiosciencesSuzhoulnc.,China), and observed and photographed under inverted microscope.

\section{Lentivirus infection}

The third generation of rabbit BMSCs, were divided into three groups according to the transfection conditions: group A (BMSCs), group B (BMSCs+Lv-EGFP) and group C (BMSCs+Lv-NMNAT3-EGFP). Lentivirus transfection (ShanghaiHengYuanBiologicalTechnologyCo.,Ltd.,China) (MOI=100) was performed for 12 hours according to the experimental group ${ }^{037 \square}$. After 3 days, the expression of green fluorescent protein was observed by inverted fluorescence microscope and the transfection efficiency was calculated. After 5 days of transfection, the culture medium containing puromycin (Solarbio,Beijing,China) $(2 \mathrm{ug} / \mathrm{mL})$ was added for screening. After all the cells in the control group died, the concentration of puromycin was halved $(1 \mathrm{ug} / \mathrm{mL})$ to obtain stable strains.

\section{Real-time RT-PCR}

Isolation and extraction of total RNA from cells using Trizol(Invitrogen,USA),take $10 \mu \mathrm{L}$ of total RNA for reverse transcription using RevertAid ${ }^{\text {tw }} 1$ st-strand cDNA Synthesis Kit (SangonBiotech,China). The reaction parameters were as follows: $65^{\circ} \mathrm{C}, 5 \mathrm{~min} ; 42^{\circ} \mathrm{C}, 30 \mathrm{~min} ; 70{ }^{\circ} \mathrm{C}, 10 \mathrm{~min} .1 \mu \mathrm{L}$ reverse transcriptional product cDNA was used for real-time fluorescence quantitative PCR, cycle parameters: 95 ${ }^{\circ} \mathrm{C}, 3 \mathrm{~min} ; 95^{\circ} \mathrm{C}, 3 \mathrm{~min} ; 60{ }^{\circ} \mathrm{C}, 30 \mathrm{~s} ; 40$ cycles. At the same time of determining the target gene, the 
endogenous butler gene ( $\beta$-actin), was determined with the internal reference as the comparative standard. Using the comparative threshold method: the relative expression quantity of the target gene $=2^{\square}$ $\triangle \triangle \mathrm{Ct}$, was converted into the relative quantitative relationship of the initial template number of the sample.Rabbit primers (Sangon Biotech) were as follows: NMNAT3-F: CCCGTCAATGACAGCTACAGGAAG; NMNAT3-R: AGCACCTTCACCGTCTCCATCC; $\beta$-actin-F: TCCCTGGAGAAGAGCTACGA; $\beta$-actin: GTACAGGT CCTTGCGGATGT.

\section{Immunoblot and Immunoprecipitation}

The total protein of each group was extracted by cell lysis, and the protein concentration of each group was determined by BCA method. After adjusting the protein concentration, the protein was boiled and denatured, and then electrophoretic, membrane transfer and sealing were carried out in turn. Than the

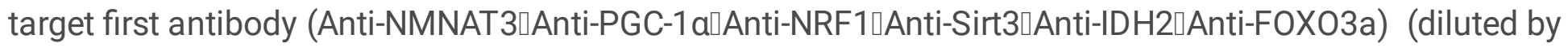
1: 300) and internal reference antibody (Anti- $\beta$-actin $\square$ Anti-COX IV) (diluted by 1: 7500) were added respectively and incubated overnight at $4{ }^{\circ} \mathrm{C}$. The TBST film was washed for 3 times, then incubated at room temperature with secondary antibody (diluted by 1: 7500) for $1 \mathrm{~h}$, and then washed for 3 times. Finally, the film was exposed by Fusion Fx exposure machine. $\beta$-actin and COX IV were used as an internal reference protein, and the relative expression of the target protein was calculated according to the gray value of protein band/ $\beta$-actin(or COX IV) protein band gray value. For co-immunoprecipitation, the cell protein was also extracted by lysate and quantified by BCA method, then the protein was added to ProteinA/G agarose beads and incubated at $4{ }^{\circ} \mathrm{C}$ for 2 hours, and the supernatant was obtained by $2500 \mathrm{rpm}$ centrifugation for $3 \mathrm{~min}$ at $4{ }^{\circ} \mathrm{C}$, and the corresponding antibody was put in the refrigerator at 4 ${ }^{\circ} \mathrm{C}$ and slowly rotated to incubate overnight. The next day, ProteinA/G agarose beads were slowly incubated at $4{ }^{\circ} \mathrm{C}$ for $3 \mathrm{~h}, 2500 \mathrm{rpm}$ centrifugation for 3 minutes, and the precipitate was slowly washed for 3 times. For the last time, carefully absorb the supernatant and add the sample buffer to the precipitation to mix well; boil at $95^{\circ} \mathrm{C}$ for $5 \mathrm{~min}$ and centrifuge at $12000 \mathrm{rpm}$ for $1 \mathrm{~min}$; Western Blot detection was carried out according to the above method.

\section{Cellular oxidative stress}

After the stable strain of BMSCs overexpressing NMNAT3 gene was obtained, according to the experimental group, each group was treated with $\mathrm{H}_{2} \mathrm{O}_{2}$ with a concentration of $600 \mu \mathrm{M}$ BMSCs for 24 hours $^{\square 35 \square}$, and the control group continued to be cultured in complete L-DMEM medium.

\section{Observation of mitochondrial ultrastructure with electron microscope}


After the BMSCs treatment of each group, routine digestion and centrifugation, EP tube to collect cells, add $3 \%$ glutaraldehyde to fix, then dehydration, osmosis, entrapment and other treatments, and make ultra-thin sections, electron staining, transmission electron microscopy to observe the ultrastructural changes of mitochondria, and select a typical visual field to take pictures.

\section{JC-1 staining}

According to the instructions of $\mathrm{JC}-1$ mitochondrial membrane potential detection kit, the treated cells were incubated with dye mixture at $37^{\circ} \mathrm{C}$ for 30 minutes and washed gently for 3 times. the cells were observed under laser confocal microscope and recorded by random typical visual field. The red / green fluorescence ratio was calculated by Image software.

\section{$\mathrm{NAD}^{+}$content detection}

According to the experimental group, the well-growing BMSCs was taken for routine digestion, the cells were suspended in the complete culture medium, the appropriate cell concentration was adjusted, and the cells were inoculated in 96-well plate to continue culture. after the cell growth density met the requirements, the operation was carried out according to the instructions of $\mathrm{NAD}^{+}$detection kit. After the cells of each group were treated, the standard curve was drawn by enzyme labeling instrument, and the absorbance at 520nm wavelength of each group was recorded and calculated.

\section{ATP levels detection}

According to the experimental group, the well-growing BMSCs was taken for routine digestion, the cells were suspended in the complete culture medium, the appropriate cell concentration was adjusted, and the cells were inoculated in 96-well plate to continue culture. after the cell growth density met the requirements, the operation was carried out according to the instructions of ATP detection kit. After the cells of each group were treated, the standard curve was drawn by enzyme labeling instrument, and the absorbance at 532nm wavelength of each group was recorded and calculated.

\section{DCFH-DA staining}

After the third generation of BMSCs was digested and centrifuged, the cell density was adjusted to $2.5 \times 10^{4} / \mathrm{mL}, 1 \mathrm{~mL} /$ dish and cultured in confocal petri dish, and then treated according to the conditions of each group. Finally, the cells in each group were treated strictly in accordance with the instructions of 
the ROS detection kit, the red fluorescence channel was detected under confocal microscope, and the pictures were collected.

\section{MDA content detection}

The third generation of BMSCs was digested and centrifuged and inoculated in 96-well plates with $2.5 \times 10^{3}$ cells per well. Then, according to the treatment conditions of each group, the cells were lysed with IP cell lysate, and the cell lysate was collected and centrifuged in EP tube to absorb the supernatant. Finally, strictly according to the instructions of the (MDA) determination kit, the absorbance value was measured and calculated at the $532 \mathrm{~nm}$ wavelength by the enzyme labeling instrument.

\section{ß-gal staining}

According to the experimental group, the cells were fixed at room temperature for 15 minutes, and the staining solution was prepared according to the cell senescence $\beta$-galactosidase staining kit. According to the instructions of the kit, after adding the working solution, the cells were cultured overnight at $37{ }^{\circ} \mathrm{C}$ and observed with an ordinary optical microscope.

\section{Cell viability and proliferation}

After routine trypsin digestion of BMSCs in each group, the complete culture medium was suspended and inoculated into 96 -well plates according to (2.0-3.0) $\times 10^{3} /$ well. There were 4 multiple holes in each group, a total of 8 plates, and continued culture in $37{ }^{\circ} \mathrm{C}$ and $5 \% \mathrm{CO}_{2}$ incubator. After that, one plate was taken at the fixed time every day according to the operation instructions of CCK-8 kit. After the cells of each group were treated, the absorbance (OD value) of each group at $450 \mathrm{~nm}$ was measured by enzyme labeling instrument, and continuously monitored for 8 days. The cell growth curve of each group was drawn with the cell culture days as the horizontal axis and the average absorbance value of each group as the vertical axis.

\section{TUNNEL/DAPI detection of apoptosis}

According to the experimental group, the treated cells were digested and centrifuged, and the cell density was adjusted to $2.5 \times 10^{4} / \mathrm{mL}, 1 \mathrm{~mL} /$ dish, then inoculated in confocal petri dish, and then treated according to each treatment condition. Finally, the cells of each group were treated strictly in accordance with the instructions of TUNEL and DAPI detection kit, observed under confocal microscope, and the pictures were collected. 


\section{Statistical analysis}

All statistical data were calculated and graphed using GraphPad Prism software version 6 (GraphPad Software, San Diego, California, USA). All numerical data are expressed as mean \pm standard deviation $(S D)( \pm S D)$. Statistical significance was evaluated using paired two-tailed Student's t-test. Differences were considered significant at $p<0.05$.

\section{Results}

\section{Overexpression of NMNAT3 in BMSCs and increased of Mitochondrial NAD+ level}

We used NMNAT3-loaded lentivirus to transfect BMSCs and selected stable strains. The mRNA expression of NMNAT3 was detected by qRT-PCR and the protein expression of NMNAT3 was evaluated by Western Blot. The results of qPCR showed that the level of mRNA in overexpression group was significantly higher than that in control group and the empty virus group $\left({ }^{\star} P<0.05\right)$. The expression was up-regulated by $(3.475 \pm 0.263)$ times, and there was no significant difference between the control group and the empty virus group (Fig. 1A). Western Blot results showed that positive protein bands with molecular weight of about 28kDa were detected in each group of BMSCs (Fig. 1B), and the expression of NMNAT3 protein in overexpression group was significantly higher than that in control group or empty virus group, and the protein content was increased approximately by $(2.844 \pm 0.152)$ times (Fig. 1C). These results confirmed the successful overexpression of NMNAT3 gene in BMSCs.

NMNAT3 is a key enzyme in the synthesis of $\mathrm{NAD}^{+}$in mitochondria and is essential for the steady-state regulation of $\mathrm{NAD}^{+}$in the mitochondria ${ }^{\square 30-34 \square}$. In order to further observe the effect of NMNAT3 overexpression on the levels of $\mathrm{NAD}^{+}$in mitochondria, according to the manufacturer's plan, WST- 8 method was used to detect the content of $\mathrm{NAD}^{+}$. The results showed that the increase trend was similar to that the expression of NMNAT3. The levels of NAD ${ }^{+}$in mitochondria of BMSCs modified with NMNAT3 gene increased significantly, about $(346.314 \pm 7.473)$ pmol/ $10^{6}$ cells, compared with the control group, it increased by $(2.066 \pm 0.385)$ times, the difference was statistically $\left({ }^{\star} P<0.05\right)$. There was no significant difference between the control group and the empty virus group (Fig. 1D).

\section{Fig. 1 Expression of NMNAT3 and the function of BMSCs after transfection}

qPCR analysis of relative expression of NMNAT3 mRNA $(n=3)$. B,C. Western blot analysis of NMNAT3 protein expression $(n=3)$. D. NAD levels detection of mitochondria $(n=3)$. E. Identification of BMSCs surface antigen by flow cytometry Note $(n=3)$. F. Observation of staining induced by multi-directional differentiation $(\mathrm{n}=3)$. G. cell proliferation curve $(\mathrm{n}=3)$. All data are presented as means $\left.\pm S D,{ }^{*} P<0.05,{ }^{\#} P\right]$ 05. BMSC, bone marrow mesenchymal stem cells; NMNAT3, nicotinamide mononucleotide adenylyl 
transferase 3; qPCR, Real-time Quantitative PCR; mRNA, messenger RNA; NAD ${ }^{+}$, nicotinamide adenine dinucleotide; CCK-8, cell counting kit-8.

\section{BMSCs overexpressing NMNAT3 retains its mesenchymal properties and proliferative ability}

We successfully obtained a stable strains of BMSCs with high expression of NMNAT3 gene by lentivirus gene transfection, but it is unknown whether the mesenchymal characteristics and proliferation of NMNAT3 modified BMSCs are affected ${ }^{\square 38}$. The surface antigens were identified by flow cytometry. The results showed that NMNAT3-modified BMSCs still expressed typical mesenchymal stem cell markers CD29, CD90 and CD106 (> 99\%), while hematopoietic CD45 markers and monocyte CD11b markers showed low expression ( $<1 \%$ ). And its level is similar to that of the control group BMSCs (Fig. 1E, Tab. I). For its multidirectional differentiation potential ${ }^{\square 39}$, we used commercial kits to induce osteogenic, chondrogenic and adipogenic differentiation, and the results were shown as by alizarin red staining, alixin blue staining and oil red O (Fig. 1F). Both the genetically modified BMSCs and the control BMSCs could differentiate into osteoblasts, chondroblasts and adipocytes.

In addition, in order to understand the effect of gene modification on the proliferation of BMSCs, we continuously cultured the cells of each group and drew the growth curve by CCK-8 method. The cells in each group showed a similar proliferation trend. After a latent adaptation period of 1-2 days, a large number of cells began to proliferate into the logarithmic growth phase on the 3rd day, maintained about 3 days logarithmic growth phase, and then entered the plateau phase (Fig. 1G). Here, our study shows that after lentivirus transfection, the proliferation of genetically modified BMSCs is not affected.

The above data show that gene modification does not change the mesenchymal characteristics of BMSCs, and still retains a high ability of proliferation and differentiation.

Tab. I Flow cytometry analysis of CD labeling of stem cells in the third generation control group (Control), empty virus group (Lv-EGFP) and NMNAT3 overexpression group (Lv-NMNAT3)

\begin{tabular}{lccc} 
Stem cell marker & Control & Lv-EGFP & Lv-NMNAT3 \\
\hline CD45 & $0.04 \%$ & $0.07 \% \triangle$ & $0.06 \%$ \\
\hline CD11b & $0.38 \%$ & $0.59 \% \triangle$ & $0.67 \%$ \\
\hline CD29 & $99.97 \%$ & $99.79 \% \triangle$ & $100.00 \%^{\square}$ \\
\hline CD90 & $100.00 \%$ & $99.99 \% \triangle$ & $100.00 \%^{\square}$ \\
\hline CD106 & $99.72 \%$ & $99.62 \% \triangle$ & $99.68 \%^{\square}$
\end{tabular}


Note: the expression of CD markers in BMSCs of each group, $n=3, \triangle P \otimes 0.05$ Vs Control, ${ }^{\square} P \otimes 0.05$ Vs LvEGFP.

\section{NMNAT3 overexpression ameliorated mitochondrial damage under oxidative stress}

The oxidative stress microenvironment in the transplantation area leads to the impairment of mitochondrial function of transplanted stem cells, which is the main cause of stress apoptosis of stem cells $^{\square 5,8-11 \square}$. The $\mathrm{H}_{2} \mathrm{O}_{2}(600 \mu \mathrm{M})$ was used to simulate the oxidative stress microenvironment ${ }^{035 \square}$. The mitochondrial ultrastructure of BMSCs was observed by transmission electron microscopy after 24 hours treated with $\mathrm{H}_{2} \mathrm{O}_{2}$. The mitochondrial ultrastructure of $\mathrm{BMSCs}$ in $\mathrm{H}_{2} \mathrm{O}_{2}$ treated group was unclear, and the mitochondrial crest fused and broken, while the ultrastructure of BMSCs in untreated group was clear and no obvious damaging change was found (Fig. 2A). The mitochondrial membrane potential of cells was measured by JC-1 method ${ }^{\square 40,410}$. Observation by laser confocal microscopy showed that the fluorescence markers of $\mathrm{H}_{2} \mathrm{O}_{2}$ treated group showed a low red and high green change, while the untreated group still showed a high red and high green. Compared with that, the mitochondrial membrane potential of cells decreased significantly in the $\mathrm{H}_{2} \mathrm{O}_{2}$ treated group (Fig. 2B,C). We used luciferase analysis to detect the ATP production of BMSCs mitochondria in each group ${ }^{\square[}$. The results showed that the mitochondrial ATP synthesis in the $\mathrm{H}_{2} \mathrm{O}_{2}$ treated group was significantly lower than that in the untreated group, Compared with the untreated group, ATP synthesis decreased by approximately $(2.232 \pm 0.372)$ times, and the difference was statistically significant $\left({ }^{*} P<0.05\right.$,Fig. 2D). In addition, the levels of $N A D^{+}$and the synthesis of mitochondrial proteins PGC-1 $\mathrm{a}$ and NRF1 in BMSCs mitochondria treated with $\mathrm{H}_{2} \mathrm{O}_{2}$ also showed the same decreasing trend. Compared with the untreated group, they respectively decreased by $(1.734 \pm 0.277)$ times, $(3.485 \pm 0.423)$ times and $(3.727 \pm 0.308)$ times, and the difference was statistically significant $\left({ }^{*} P<0.05\right.$, Fig. $\left.2 \mathrm{E}-\mathrm{H}\right)$. The above results indicate that the structure and function of BMSCs mitochondria are significantly impaired under oxidative stress.

In order to further study the effect of NMNAT3 on the mitochondrial function of BMSCs under oxidative stress, we continued to use $\mathrm{H}_{2} \mathrm{O}_{2}$ to treat BMSCs in NMNAT3 modified. It was observed by transmission electron microscopy that the ultrastructure of mitochondria in the overexpression group was still clear, the mitochondrial crest was continuous, and no obvious damage changes were observed (Fig. 2A). For the mitochondrial membrane potential, the immunofluorescence of BMSCs in the overexpression group showed high red and green, and there was no significant difference compared with the untreated group, but the mitochondrial membrane potential was significantly higher than that in the control group and empty virus group treated with $\mathrm{H}_{2} \mathrm{O}_{2}$, and the difference was statistically significant $\left({ }^{*} P<0.05, \mathrm{Fig} .2 \mathrm{~B}, \mathrm{C}\right)$. In addition, NMNAT3 modified BMSCs showed higher levels of NAD ${ }^{+}$, ATP, PGC-1a and NRF1 synthesis, which were $(272.214 \pm 7.068) \mathrm{pmol} / 10^{6}$ cells, $(9.774 \pm 0.523) \mathrm{nmol} / \mathrm{mg},(2.255 \pm 0.216)$ times and $(2.427 \pm 0.174)$ times respectively. Compared with the control group and empty virus group, the mitochondrial function of BMSCs in overexpression group was significantly improved $\left({ }^{*} P<0.05\right.$, Fig. $2 D$ - 
H). In summary, these results suggest that NMNAT3 gene overexpression can effectively improve the damage of mitochondrial structure and functions of BMSCs under oxidative stress.

\section{Fig. 2 NMNAT3 improves mitochondrial function under oxidative stress}

Observation of ultrastructure of mitochondria by transmission electron microscope $(n=3)$. B,C. Detection of mitochondrial membrane potential by JC-1 kit $(n=3)$. D. ATP levels detection of mitochondria $(n=3)$. E. $N A D^{+}$levels detection of mitochondria $(n=3)$. F-H. Western blot analysis of PGC-1a and NRF1 expression in mitochondria $(n=3)$. All data are presented as means $\pm S D,{ }^{*} P<0.05,{ }^{\#} P \otimes 05$. JC-1, 5,5',6,6'-Tetrachloro1,1',3,3'-tetraethyl-imidacarbocyanine; ATP, Adenosine triphosphate; PGC-1a, Peroxisome proliferatoractivated receptor gamma coactivator-1a; NRF1, nuclear respiratory factors 1; COX IV, Cytochrome c oxidase IV.

\section{The anti-oxidative stress ability of BMSCs modified by NMNAT3 is enhanced}

NMNAT3 modified BMSCs can effectively improve the function of mitochondria under oxidative stress, but it is not clear whether the antioxidant stress ability of modified BMSCs is enhanced. We analyzed the basic antioxidant capacity of BMSCs, and detected the level of ROS, the content of MDA and the activities of antioxidant enzymes CAT and GPX in BMSCs respectively ${ }^{\llbracket 42,43 \square}$. As shown in figure 3, compared with the control group, the intracellular oxidative damage indicators ROS levels and MDA contents of NMNAT3 gene modified BMSCs decreased significantly under $\mathrm{H}_{2} \mathrm{O}_{2}$ simulated oxidative stress conditions, about $(5.656 \pm 0.239)$ times and $(2.664 \pm 0.185)$ times, while the activities of antioxidant enzymes CAT and GPX increased significantly, respectively $(11.365 \pm 0.577) \mathrm{U} / \mathrm{mg}$ and $(14.629 \pm 0.729)$ $\mathrm{U} / \mathrm{mg}$, the difference was statistically significant $\left({ }^{\star} P<0.05\right.$, Fig. $\left.3 A-E\right)$. In addition, the senescence-related SA- $\beta-G$ al activity and apoptosis in BMSCs of each group were also detected ${ }^{\square 44}$. In the NMNAT3 overexpression group, the SA- $\beta-G$ al activity decreased, the percentage of SA- $\beta-G$ al positive cells decreased by $(3.152 \pm 0.756)$ times, and the number of apoptotic cells also decreased significantly, while the control group and empty virus group under the same conditions did not show the same change trend, the difference was statistically significant $\left({ }^{*} P<0.05\right.$, Fig. $\left.3 \mathrm{~F}-\mathrm{H}\right)$. The above results showed that the antioxidant stress ability of BMSCs modified by NMNAT3 was enhanced.

\section{Fig. 3 NMNAT3 overexpression enhances the antioxidant stress ability of BMSCs}

A,B. DCFH-DA fluorescence probe detection of ROS $(n=3)$. C. MDA in BMSCs Content detection. D. Analysis of CAT activity in BMSCs. E. Analysis of GPx activity in BMSCs. F,G. Detection of $\beta$-gal activity by $\beta$-gal staining $(n=4)$. H. TUNEL/DAPI staining method for detecting apoptosis $(n=4)$. All data are presented as means $\pm S D,{ }^{*} P<0.05,{ }^{\#} P \otimes 0.05$. DCFH-DA, 2',7'-Dichlorofluorescin diacetate; ROS, reactive oxygen species; MDA, Malondialdehyde; CAT, Catalase; GPx, Glutathione peroxidase; SA- $\beta-G a l$, senescence associated- $\beta$-galactosidase; TUNEL, terminal deoxynucleotidyl transferase dUTP nick end labeling; DAPI, 4',6-diamidino-2-phenylindole. 


\section{Overexpression of NMNAT3 enhances the activity of Sirt3 under oxidative stress}

At the molecular level, the mechanism by which NMNAT3 overexpression modified BMSCs and increases $\mathrm{NAD}^{+}$levels in mitochondria to improve mitochondrial function and enhance its antioxidant stress ability is not clear. Sirt3 is an important protein that regulates mitochondrial metabolism and oxidative homeostasis, and its activity is closely related to the levels of $N A D^{+\square 26,45,46 \square}$. Does the decrease of NAD levels caused by mitochondrial dysfunction under oxidative stress affect Sirt3 catalytic activity, limit its regulation and improve mitochondrial function? By increasing the expression of NMNAT3 and increasing the levels of $\mathrm{NAD}^{+}$, does it affect the catalytic activity of Sirt3, and then improve the mitochondrial function? Therefore, we quantitatively analyzed the expression and activity of Sirt3 by Western Blot and colorimetry. The expression levels of Sirt3 in BMSCs of NMNAT3 overexpression group was not significantly increased, but its activity was significantly higher than that of the control group and empty virus group, approximately $(1.545 \pm 0.073)$ times. $\left({ }^{*} P<0.05\right.$, Fig. $\left.4 \mathrm{~A}, \mathrm{~B}\right)$. Further detection by Co-IP method showed that, compared with the empty virus group, the acetylation levels of Idh2 and FOXO3a in BMSCs modified by NMNAT3 gene decreased by $(2.746 \pm 0.117)$ times and $(2.625 \pm 0.146)$ times respectively, the activities of ATP synthase and Mn-SOD increased by $(1.551 \pm 0.098)$ times and $(1.829 \pm 0.083)$ times, and the ratio of GSH/GSSH increased by $(1.715 \pm 0.139)$ times, and all differences were statistically significant $\left({ }^{*} P<0.05\right.$, Fig. $\left.4 A, C-G\right)$. These results indicate that NMNAT3 increases the levels of $N A D^{+}$in mitochondria and plays a role in the activation of Sirt3. However, we are not sure that the abovfresulte changes are NAD ${ }^{+}$-Sirt3 dependent, and we can not rule out that it may be caused by the direct regulation of NMNAT3$\mathrm{NAD}^{+}$. Therefore, we further used the selective Sirt3 inhibitor (3-TYP) to inhibit the activity of Sirt $3^{\square 47 \square}$. The results showed that the addition of Sirt3 inhibitors increased the acetylation levels of Idh2 and FOXO3a in mitochondria of NMNAT3 modified BMSCs, while the levels of GSH, ATP synthase and Mn-SOD activity decreased, and the effect of NMNAT3 was completely blocked (Fig. 4A-G). Here, our results further support that NMNAT3 through the NAD ${ }^{+}$-Sirt3 axis is a potential mechanism for improving mitochondrial function and enhancing the antioxidant stress ability of BMSCs.

\section{Fig.4 NMNAT3 enhances the activity of Sirt3 under oxidative stress}

A,C,D. Western blot analysis of Sirt3, IDH2 and FOXO3a levels in BMSCs. Acetylated IDH2 and FOXO3a were isolated by immunoprecipitation with anti-IDH2 and anti-FOXO3a antibody followed by western blotting with anti-acetyl-lysine antibody $(n=3)$. B. Detection of Sirt3 relative activity in BMSCs. E. Analysis of ATP synthase activity in BMSCs. F. Analysis of Mn-SOD activity in BMSCs. G. Detection the ratio of GSH/GSSH in BMSCs. All data are presented as means \pm SD, ${ }^{*} P<0.05,{ }^{\#} P \otimes 0.05$. Sirt3, silent mating type information regulation 2 homolog 3; Idh2, isocitrate dehydrogenase 2; FOXO3a, Forkhead-box protein O3a; Mn-SOD, manganese superoxide dismutase; GSH/GSSH, reduced glutathione/ oxidized glutathione. 


\section{Discussion}

The low survival rate of stem cell transplantation in vivo is related to the dysfunction of stem cell mitochondria caused by oxidative stress microenvironment in the transplantation area, which is mainly

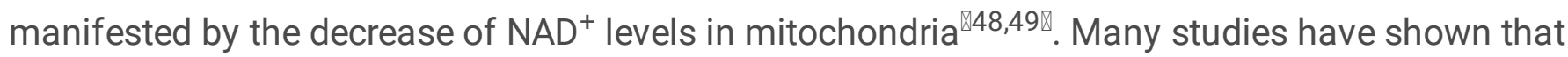
decreased $N A D^{+}$levels can inhibit the activity of $\mathrm{NAD}^{+}$-dependent enzymes in mitochondrial oxidative phosphorylation, TCA cycle and glycolysis, thereby reducing the production of ATP and damage the

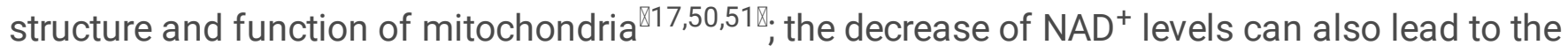
disturbance of cell signal molecular pathways, resulting in cell aging and apoptosis ${ }^{\bigotimes 14,52 \rrbracket}$. Therefore, maintaining $\mathrm{NAD}^{+}$homeostasis is an effective strategy to combat mitochondrial disorders and improve

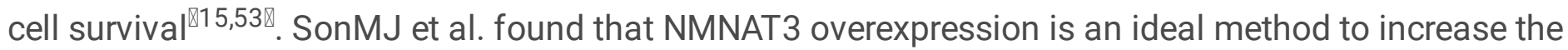
levels of $\mathrm{NAD}^{+}$in cell mitochondria ${ }^{\mathrm{B3}}$. In this study, we also found that the $N A D^{+}$levels in mitochondria of BMSCs modified by NMNAT3 was significantly higher than the control group $(2.895 \pm 0.413$ times, $\mathrm{P}<$ $0.05)$, and the decrease of $\mathrm{NAD}^{+}$levels caused by oxidative stress was recovered. It also effectively ameliorated the mitochondrial structural damage of BMSCs under oxidative stress, increased mitochondrial membrane potential, increased ATP and mitochondrial protein synthesis (Fig. 2). Compared with the control group, the mitochondrial structure and function of BMSCs modified by NMNAT3 were significantly improved, which indicated that under oxidative stress, NMNAT3 played a beneficial role in improving the damage of mitochondrial structure and function by restoring the levels of $\mathrm{NAD}^{+}$in mitochondria. In addition, under oxidative stress, the activities of antioxidant enzymes (CAT and GPx) in BMSCs modified by NMNAT3 were significantly higher than that in the control group $(P<0.05)$, the content of MDA and ROS reduced significantly (Fig. 3), which delayed cell aging and reduced apoptosis. This result can be attributed to the improvement of their ability of antioxidant stress.

Here, we also provide molecular insights into how $\mathrm{NAD}^{+}$regulates mitochondrial function. In recent years, the deacetylase Sirtuins protein family has been widely concerned by researchers ${ }^{\varangle 47,54-56 \rrbracket}$, in which Sirt3 plays an indispensable role in regulating mitochondrial energy metabolism and oxidative stress 845,46,57-59凹. The biological activity of Sirt3 depends on the levels of $N A D^{+}$, and $N A D^{+}$levels decrease during mitochondrial dysfunction, which seriously affects the activity of Sirt3 and limits its role in regulating mitochondrial function ${ }^{\varangle 26,28,60-63 \rrbracket}$. In this study, it was found that under oxidative stress, the expression of Sirt3 protein did not change significantly, but significant differences in its activity, which may be related to low NAD ${ }^{+}$levels, While BMSCs modified by NMNAT3 overexpression can significantly increase Sirt3 activity after increasing NAD ${ }^{+}$levels $(1.545 \pm 0.073$ times, $P<0.05)$, and significantly decrease the acetylation level of Sirt3-dependent deacetylation-related proteins (Idh2, FOXO3a) in mitochondria. The content and activity of effector proteases (GSH, ATP synthase, Mn-SOD) were increased (Fig. 4), which shows that under oxidative stress conditions, NMNAT3 significantly increased Sirt3 activity and improved the function of mitochondria by increasing the levels of NAD ${ }^{+}$. In order to further demonstrate the key role of Sirt3 in the effect of mitochondrial function, we treated with Sirt3 inhibitor and completely blocked this effect. The NMNAT3-NAD ${ }^{+}$did not directly decrease the acetylation 
levels of mitochondrial proteins (Idh2, FOXO3a) or increase the activity of effector proteases (GSH, ATP synthase, Mn-SOD) (Fig. 4). The results show that this change is not directly affected by NMNAT3-NAD ${ }^{+}$, but NAD+-Sirt3 is dependent. Our results support that the NMNAT3-NAD+-Sirt3 axis is a potential mechanism to improve mitochondrial function and enhance anti-oxidant stress in BMSCs.

\section{Conclusions}

In summary, We have demonstrated that the NMNAT3 gene has a positive effect on improving mitochondrial dysfunction of BMSCs under oxidative stress and enhancing the ability of antioxidant stress in vitro cell experiments, and proved that NMNAT3-NAD+-Sirt3 axis is the potential mechanism of improving mitochondrial function. This study provides a new strategy to solve the problem of survival rate of stem cell transplantation and has great significance to promote the clinical transformation of stem cell therapy. However, it is unclear whether NMNAT3 has the same effect on the complex environment in vivo ${ }^{864,65 \rrbracket}$. Future research may focus on this issue to further evaluate the role of NMNAT3 in vivo and provide experimental evidence for comprehensively elucidating the clinical application of NMNAT3.

\section{Abbreviations}

3-TYP

3-(1H-1,2,3-triazol-4-yl) pyridine

ATP

Adenosine triphosphate

BMSCs

bone marrow mesenchymal stem cells

CAT

Catalase

CCK-8

cell counting kit-8

COX IV

Cytochrome c oxidase IV

DCFH-DA

2',7'-Dichlorofluorescin diacetate

DAPI

4',6-diamidino-2-phenylindole

FOXO3a

Forkhead-box protein 03a

GPx

Glutathione peroxidase

GSH/GSSH 
reduced glutathione/oxidized glutathione

Idh2

isocitrate dehydrogenase 2

JC-1

5,5',6,6'-Tetrachloro-1,1',3,3'-tetraethyl-imidacarbocyanine

MDA

Malondialdehyde

Mn-SOD

manganese superoxide dismutase

mRNA

messenger RNA

NMNAT3

nicotinamide mononucleotide adenylyl transferase 3

$\mathrm{NAD}^{+}$

nicotinamide adenine dinucleotide

NRF1

nuclear respiratory factors 1

PGC-1a

Peroxisome proliferator-activated receptor gamma coactivator-1a

ROS

reactive oxygen species

RT-qPCR

Real-time Quantitative PCR

Sirt3

silent mating type information regulation 2 homolog 3

SA- $\beta$-Gal

senescence associated- $\beta$-galactosidase

TUNEL

terminal deoxynucleotidyl transferase dUTP nick end labeling

\section{Declarations}

\section{Acknowledgments}

Not applicable.

\section{Funding}


the Regional Science Foundation of National Natural Science Foundation of China (Grant Nos. 81860387); the Youth Science Foundation of National Natural Science Foundation of China (Grant Nos. 81902226); the Guizhou Provincial Science and Technology Department-Guizhou Medical University Joint Fund (Grant Nos. [2017]7197); the Science and Technology Department of Guiyang (Grant Nos. GY2016-3); the Science and Technology Fund of Guizhou Provincial Department of Health (Grant Nos. gzwjkj2019-1-135)

\section{Author information}

Tao Wang and Fei Zhang contributed equally to this work.

\section{Affiliations}

Clinical Medical College, Medical University of Guizhou, Guiyang, Guizhou 550004, China Tao Wang, Wuxun Peng, Fei Zhang, Lei Wang, Jian Zhang, Wentao Dong, Chuan Ye \& Xiaobin Tian

Department of Traumatology, Affiliated Hospital of Guizhou Medical University, Guiyang, Guizhou 550004, China

Wuxun Peng, Fei Zhang, Jian Zhang \& Wentao Dong

Department of Statistics, Guizhou Maternal and Child Health Hospital, Guiyang, Guizhou 550004, China Lei Wang

Department of Orthopaedics, Affiliated Hospital of Guizhou Medical University, Guiyang, Guizhou 550004, China

Chuan Ye \& Xiaobin Tian

Department of Orthopaedics, First Affiliated Hospital of Kunming Medical University, Kunming, Yunnan 650032, China 


\section{Contributions}

,W.X.P. and F.Z. generated the hypothesis and concept of the manuscript,T.W. and F.Z. designed experiments, performed most of the experiments.T.W. and J.Z. performed the mitochondrial studies,T.W.,F.Z. and W.T.D. performed cell experiments, western-blots, and PCRs.L.W. and C.Y. analyzed and interpreted the data, T.W. edited and wrote the manuscript. X.B.T., Y.L.L and Y.K.G. revised the manuscript.All authors contributed to writing the manuscript.

\section{Corresponding author}

Correspondence to Wuxun Peng.

\section{Ethics declarations}

\section{Ethics approval and consent to participate}

All animals are approved by the Experimental Animal Bioethics Committee of Guizhou Medical University (Grant No.1900590), and all procedures are carried out in strict accordance with the guidelines for the Care and use of Experimental Animals issued by the National Institutes of Health (NIH publication No. 8523, 1996 revised).

\section{Consent for publication}

Not applicable.

\section{Competing interests}

The authors declare that they have no competing interests.

\section{Availability of data and materials}

The datasets used and/or analysed during the current study are available from the corresponding author on reasonable request. 


\section{References}

1. Lee S, Choi E, Cha MJ, Hwang KC. Cell adhesion and long-term survival of transplanted mesenchymal stem cells: a prerequisite for cell therapy.Oxid Med Cell Longev. 2015; 2015: 632902.

2. Karpov A A, Udalova D V, Pliss M G, et al. Can the outcomes of mesenchymal stem cell-based therapy for myocardial infarction be improved? Providing weapons and armour to cells. Cell proliferation. 2017; 50(2): e12316.

3. Zhao L, Hu C, Zhang P, et al. Preconditioning strategies for improving the survival rate and paracrine ability of mesenchymal stem cells in acute kidney injury. J Cell Mol Med. 2019; 23(2): 720-730.

4. Karantalis V, Hare J M. Use of mesenchymal stem cells for therapy of cardiac disease. Circulation research. 2015; 116(8): 1413-1430.

5. Denu R A, Hematti P. Effects of oxidative stress on mesenchymal stem cell biology. Oxidative Medicine and Cellular Longevity. 2016; 2016.

6. Hu XF, Wang L, Xiang G, Lei W, Feng YF. Angiogenesis impairment by the NADPH oxidase-triggered oxidative stress at the bone-implant interface: critical mechanisms and therapeutic targets for implant failure under hyperglycemic conditions in diabetes. Acta Biomater. 2018;73:470-487.

7. Salazar-Noratto G E, Luo G, Denoeud C, et al. Concise Review: Understanding and leveraging cell metabolism to Enhance Mesenchymal Stem Cell Transplantation Survival in Tissue Engineering and Regenerative Medicine Applications. Stem Cells. 2019.

8. Scheibye-Knudsen M, Fang E F, Croteau D L, et al. Protecting the mitochondrial powerhouse. Trends in cell biology. 2015; 25(3): 158-170.

9. Bhatti J S, Bhatti G K, Reddy P H. Mitochondrial dysfunction and oxidative stress in metabolic disorders-A step towards mitochondria based therapeutic strategies. Biochimica et Biophysica Acta (BBA)-Molecular Basis of Disease. 2017; 1863(5): 1066-1077.

10. Hu C, Zhao L, Peng C, et al. Regulation of the mitochondrial reactive oxygen species: Strategies to control mesenchymal stem cell fates ex vivo and in vivo. Journal of cellular and molecular medicine. 2018; 22(11): 5196-5207.4.658.

11. Filippi MD, Ghaffari S. Mitochondria in the maintenance of hematopoietic stem cells: new perspectives and opportunities. Blood. 2019;133(18):1943-1952.

12. Poljsak B, Milisav I. NAD ${ }^{+}$as the link between oxidative stress, inflammation, caloric restriction, exercise, DNA repair, longevity, and health span. Rejuvenation research. 2016; 19(5): 406-413.

13. Rajman L, Chwalek K, Sinclair DA. Therapeutic Potential of NAD-Boosting Molecules: The In Vivo Evidence. Cell Metab. 2018;27(3):529-547.

14. Okabe K, Yaku K, Tobe K, Nakagawa T. Implications of altered NAD metabolism in metabolic disorders. J Biomed Sci. 2019;26(1):34 
15. Imai S, Guarente L. NAD ${ }^{+}$and sirtuins in aging and disease. Trends in cell biology. 2014; 24(8): 464471.

16. Dai S H, Chen T, Wang Y H, et al. Sirt3 protects cortical neurons against oxidative stress via regulating mitochondrial $\mathrm{Ca}^{2+}$ and mitochondrial biogenesis. International journal of molecular sciences. 2014; 15(8): 14591-14609.

17. Canto $\mathrm{C}$, Menzies $\mathrm{K}$ J, Auwerx J. NAD ${ }^{+}$metabolism and the control of energy homeostasis: a balancing act between mitochondria and the nucleus. Cell metabolism. 2015; 22(1): 31-53.

18. Jokinen R, Pirnes-Karhu S, Pietiläinen $\mathrm{K} \mathrm{H}$, et al. Adipose tissue $\mathrm{NAD}^{+}$-homeostasis, sirtuins and poly (ADP-ribose) polymerases-important players in mitochondrial metabolism and metabolic health. Redox biology. 2017; 12: 246-263.

19. Carrico C, Meyer JG, He W, Gibson BW, Verdin E. The Mitochondrial Acylome Emerges: Proteomics, Regulation by Sirtuins, and Metabolic and Disease Implications. Cell Metab. 2018;27(3):497-512.

20. Zhang X, Ji R, Liao X, et al. MicroRNA-195 regulates metabolism in failing myocardium via alterations in sirtuin 3 expression and mitochondrial protein acetylation. Circulation. 2018; 137(19): 2052-2067.

21. Hirschey M D, Shimazu T, Jing E, et al. SIRT3 deficiency and mitochondrial protein hyperacetylation accelerate the development of the metabolic syndrome. Molecular cell. 2011; 44(2): 177-190.

22. Weir HJ, Lane JD, Balthasar N. SIRT3: A Central Regulator of Mitochondrial Adaptation in Health and Disease. Genes Cancer. 2013;4(3-4):118-24.

23. Ansari A, Rahman M S, Saha S K, et al. Function of the SIRT 3 mitochondrial deacetylase in cellular physiology, cancer, and neurodegenerative disease. Aging cell. 2017; 16(1): 4-16.

24. Wang T, Cao Y, Zheng Q, et al. SENP1-Sirt3 Signaling Controls Mitochondrial Protein Acetylation and Metabolism. Mol Cell. 2019;75(4):823-834.

25. Cantó $\mathrm{C}$, Houtkooper $\mathrm{R} H$, Pirinen $\mathrm{E}$, et al. The $N A D^{+}$precursor nicotinamide riboside enhances oxidative metabolism and protects against high-fat diet-induced obesity. Cell metabolism. 2012; 15(6): 838-847.

26. Camacho-Pereira J, Tarragó M G, Chini C C S, et al. CD38 dictates age-related NAD decline and mitochondrial dysfunction through an SIRT3-dependent mechanism. Cell metabolism. 2016; 23(6): 1127-1139.

27. Hershberger K A, Martin A S, Hirschey M D. Role of $\mathrm{NAD}^{+}$and mitochondrial sirtuins in cardiac and renal diseases. Nature Reviews Nephrology. 2017; 13(4):213-225.

28. Zhang $\mathrm{M}$, Ying W. $\mathrm{NAD}^{+}$deficiency is a common central pathological factor of a number of diseases and aging: mechanisms and therapeutic implications. Antioxidants \& redox signaling. 2019; 30(6): 890-905.

29. Lin J B, Apte R S. NAD ${ }^{+}$and sirtuins in retinal degenerative diseases: A look at future therapies. Progress in retinal and eye research. 2018; 67: 118-129. 
30. Felici R, Lapucci A, Ramazzotti M, et al. Insight into molecular and functional properties of NMNAT3 reveals new hints of NAD homeostasis within human mitochondria. PloS one. 2013; 8(10):e76938.

31. N Jayaram H, Kusumanchi P, A Yalowitz J. NMNAT expression and its relation to NAD metabolism. Current medicinal chemistry. 2011; 18(13): 1962-1972.

32. VanLinden M R, Dölle C, Pettersen I K N, et al. Subcellular distribution of NAD ${ }^{+}$between cytosol and mitochondria determines the metabolic profile of human cells. Journal of Biological Chemistry. 2015; 290(46): 27644-27659.

33. Son MJ, Kwon Y, Son T, Cho YS. Restoration of Mitochondrial NAD+ Levels Delays Stem Cell Senescence and Facilitates Reprogramming of Aged Somatic Cells. Stem Cells. 2016;34(12):28402851.

34. Gulshan M, Yaku K, Okabe K, et al. Overexpression of Nmnat3 efficiently increases NAD and NGD levels and ameliorates age-associated insulin resistance. Aging cell. 2018; 17(4): e12798.

35. Chen M, Chen S, Lin D. Carvedilol protects bone marrow stem cells against hydrogen peroxideinduced cell death via PI3K-AKT pathway. Biomedicine \& Pharmacotherapy. 2016; 78: 257-263.

36. Tan S L, Ahmad T S, Selvaratnam L, et al. Isolation, characterization and the multi-lineage differentiation potential of rabbit bone marrow-derived mesenchymal stem cells. Journal of anatomy. 2013; 222(4): 437-450.

37. Peng W, Zhang J, Zhang H, Liu G, Dong W, Zhang F. Effects of lentiviral transfection containing bFGF gene on the biological characteristics of rabbit BMSCs. J Cell Biochem. 2018;119(10):8389-8397.

38. Dominici M, Le Blanc K, Mueller I, et al. Minimal criteria for defining multipotent mesenchymal stromal cells. The International Society for Cellular Therapy position statement. Cytotherapy. 2006; 8(4): 315-317.

39. Jiang $Y$, Jahagirdar BN, Reinhardt RL, et al. Pluripotency of mesenchymal stem cells derived from adult marrow. Nature. 2002;418(6893):41-9.

40. Zorova L D, Popkov V A, Plotnikov E Y, et al. Mitochondrial membrane potential. Analytical biochemistry. 2018; 552: 50-59.

41. Nicholls DG. Fluorescence Measurement of Mitochondrial Membrane Potential Changes in Cultured Cells. Methods Mol Biol. 2018;1782:121-135.

42. Del Rio D, Stewart A J, Pellegrini N. A review of recent studies on malondialdehyde as toxic molecule and biological marker of oxidative stress. Nutrition, metabolism and cardiovascular diseases. 2005; 15(4): 316-328.

43. Walker C L, Pomatto L C D, Tripathi D N, et al. Redox regulation of homeostasis and proteostasis in peroxisomes. Physiological reviews. 2017; 98(1): 89-115.

44. Correia-Melo $C$, et al. Mitochondria are required for pro-ageing features of the senescent phenotype. EMBO J. 2016; 35:724-742.

45. Hirschey MD, Shimazu T, Goetzman E, et al. SIRT3 regulates mitochondrial fatty-acid oxidation by reversible enzyme deacetylation. Nature. 2010; 464(7285):121-125. 
46. Bause A S, Haigis M C. SIRT3 regulation of mitochondrial oxidative stress. Experimental gerontology. 2013; 48(7): 634-639.

47. Zhai M, Li B, Duan W, et al. Melatonin ameliorates myocardial ischemia reperfusion injury through SIRT3-dependent regulation of oxidative stress and apoptosis. J Pineal Res. 2017; 63(2).

48. Zhang $H$, Ryu $D, W u Y$, et al. $N A D^{+}$repletion improves mitochondrial and stem cell function and enhances life span in mice. Science. 2016; 352(6292): 1436-1443.

49. Zhang $\mathrm{H}$, Menzies $\mathrm{K} \mathrm{J}$, Auwerx $\mathrm{J}$. The role of mitochondria in stem cell fate and aging. Development. 2018; 145(8): dev143420.

50. Stein LR, Imai S. The dynamic regulation of NAD metabolism in mitochondria. Trends Endocrinol Metab. 2012; 23(9):420-8.

51. Cantó C, Menzies KJ, Auwerx J. NAD ${ }^{+}$Metabolism and the Control of Energy Homeostasis: A Balancing Act between Mitochondria and the Nucleus. Cell Metab. 2015; 22(1):31-53.

52. Fang $E F$, Hou $Y$, Lautrup $S$, et al. $N A D^{+}$augmentation restores mitophagy and limits accelerated aging in Werner syndrome. Nat Commun. 2019; 10(1):5284.

53. Cantó $\mathrm{C}$, Houtkooper $\mathrm{RH}$, Pirinen $\mathrm{E}$, et al. The $\mathrm{NAD}^{+}$precursor nicotinamide riboside enhances oxidative metabolism and protects against high-fat diet-induced obesity. Cell Metab. 2012; 15(6):838-47.

54. Kumar S, Lombard DB. Mitochondrial sirtuins and their relationships with metabolic disease and cancer. Antioxid Redox Signal. 2015; 22(12):1060-77.

55. Hershberger K A, Martin A S, Hirschey M D. Role of $\mathrm{NAD}^{+}$and mitochondrial sirtuins in cardiac and renal diseases. Nature Reviews Nephrology. 2017; 13(4): 213-225.

56. Carrico C, Meyer J G, He W, et al. The mitochondrial acylome emerges: proteomics, regulation by sirtuins, and metabolic and disease implications. Cell metabolism. 2018; 27(3): 497-512.

57. Jing E, Emanuelli B, Hirschey M D, et al. Sirtuin-3 (Sirt3) regulates skeletal muscle metabolism and insulin signaling via altered mitochondrial oxidation and reactive oxygen species production. Proceedings of the national academy of sciences. 2011; 108(35): 14608-14613.

58. Morigi M, Perico L, Rota C, et al. Sirtuin 3-dependent mitochondrial dynamic improvements protect against acute kidney injury. J Clin Invest. 2015; 125(2):715-26.

59. Singh C K, Chhabra G, Ndiaye M A, et al. The role of sirtuins in antioxidant and redox signaling. Antioxidants \& redox signaling. 2018; 28(8): 643-661.

60. Nogueiras R, Habegger K M, Chaudhary N, et al. Sirtuin 1 and sirtuin 3: physiological modulators of metabolism. Physiological reviews. 2012; 92(3): 1479-1514.

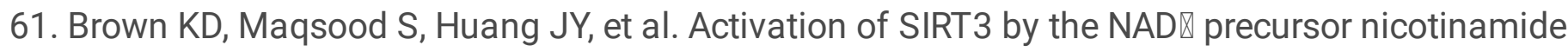
riboside protects from noise-induced hearing loss. Cell Metab. 2014; 20(6):1059-68.

62. Yamaguchi S, Yoshino J. The pathophysiological importance and therapeutic potential of NAD' biosynthesis and mitochondrial sirtuin SIRT3 in age-associated diseases. Nihon Rinsho. 2016; 74(9):1447-1455. 
63. Zhang N, Sauve A A. Regulatory effects of $\mathrm{NAD}^{+}$metabolic pathways on sirtuin activity. Prog Mol Biol Transl Sci. 2018; 154: 71-104.

64. Si Y L, Zhao Y L, Hao H J, et al. MSCs: biological characteristics, clinical applications and their outstanding concerns. Ageing research reviews. 2011;10(1): 93-103.

65. Galderisi U, Giordano A. The gap between the physiological and therapeutic roles of mesenchymal stem cells. Medicinal research reviews. 2014; 34(5): 1100-1126.

\section{Figures}
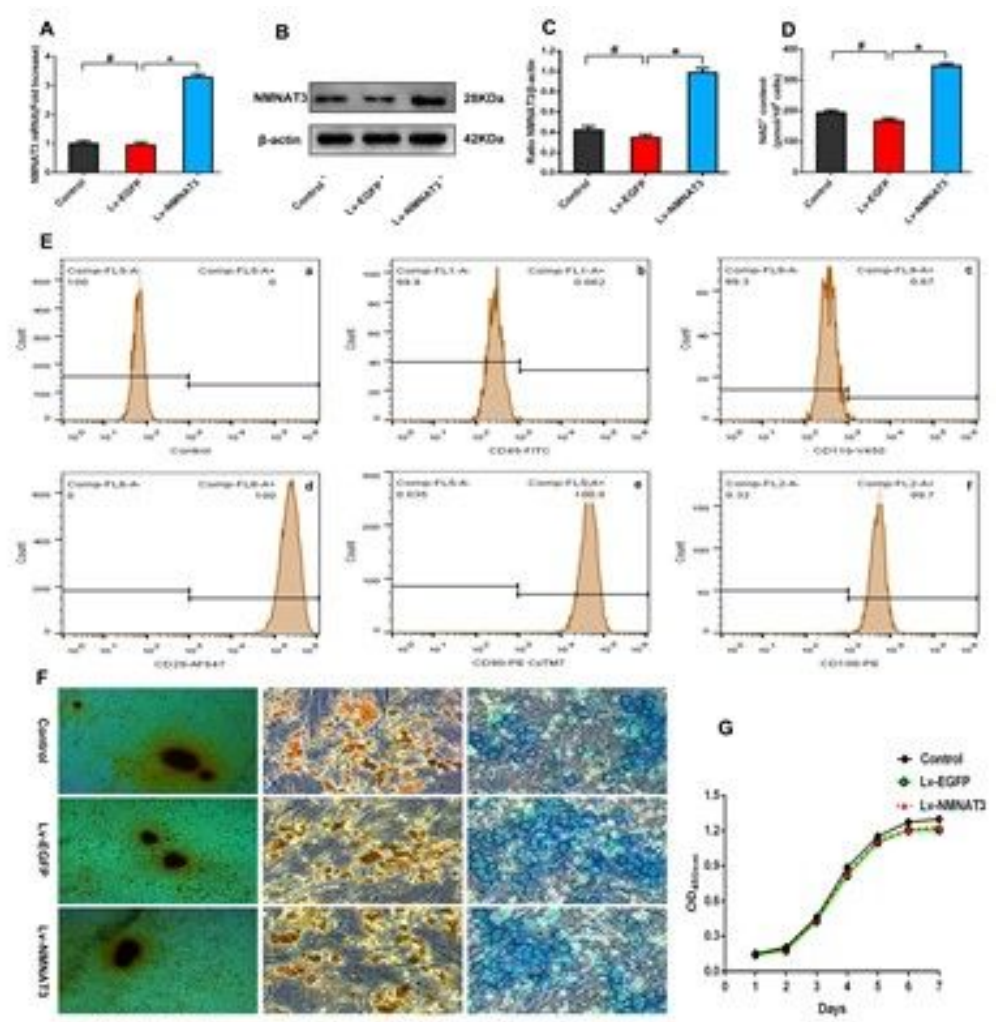

\section{Figure 1}

Expression of NMNAT3 and the function of BMSCs after transfection A. qPCR analysis of relative expression of NMNAT3 mRNA $(n=3)$. B,C. Western blot analysis of NMNAT3 protein expression $(n=3)$. D. $N A D+$ levels detection of mitochondria $(n=3)$. E. Identification of BMSCs surface antigen by flow cytometry Note $(n=3)$. F. Observation of staining induced by multi-directional differentiation $(n=3)$. G. cell proliferation curve $(n=3)$. All data are presented as means $\pm S D, * P<0.05, \# P \otimes 0.05$. BMSC, bone marrow mesenchymal stem cells; NMNAT3, nicotinamide mononucleotide adenylyl transferase 3; qPCR, Real-time Quantitative PCR; mRNA, messenger RNA; NAD+, nicotinamide adenine dinucleotide; CCK-8, cell counting kit-8. 

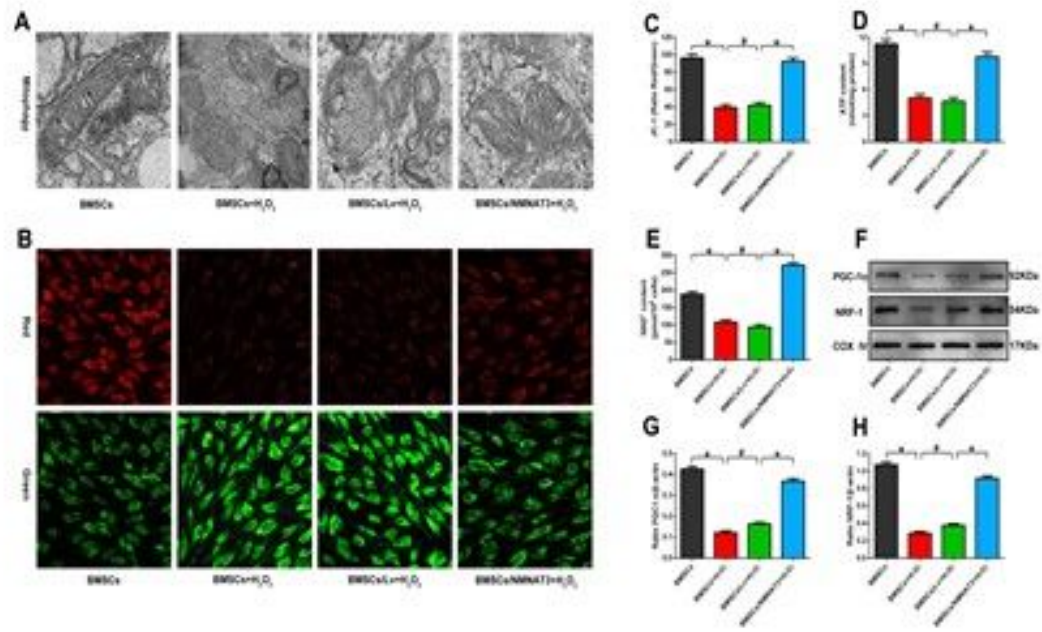

Figure 2

NMNAT3 improves mitochondrial function under oxidative stress A. Observation of ultrastructure of mitochondria by transmission electron microscope $(n=3)$. B,C. Detection of mitochondrial membrane potential by JC-1 kit $(n=3)$. D. ATP levels detection of mitochondria $(n=3)$. E. NAD+ levels detection of mitochondria $(n=3)$. F-H. Western blot analysis of PGC-1a and NRF1 expression in mitochondria $(n=3)$. All data are presented as means $\pm S D, * P<0.05$, \#Pख0.05. JC-1, 5,5',6,6'-Tetrachloro-1,1',3,3'-tetraethylimidacarbocyanine; ATP, Adenosine triphosphate; PGC-1a, Peroxisome proliferator-activated receptor gamma coactivator-1a; NRF1, nuclear respiratory factors 1; COX IV, Cytochrome c oxidase IV. 


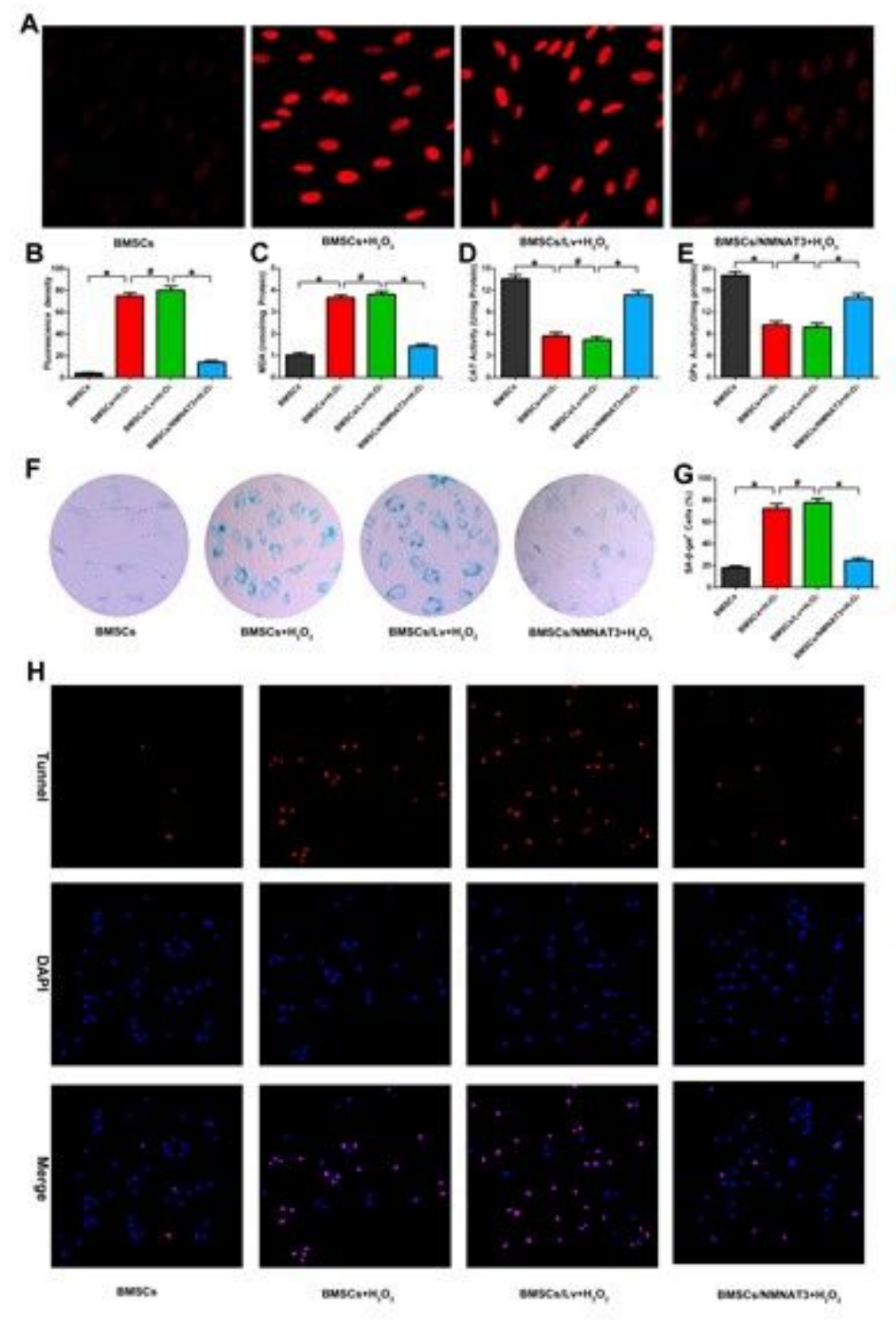

\section{Figure 3}

NMNAT3 overexpression enhances the antioxidant stress ability of BMSCs A,B. DCFH-DA fluorescence probe detection of ROS $(n=3)$. C. MDA in BMSCs Content detection. D. Analysis of CAT activity in BMSCs. E. Analysis of GPx activity in BMSCs. F,G. Detection of $\beta$-gal activity by $\beta$-gal staining $(n=4)$. $H$. TUNEL/DAPI staining method for detecting apoptosis $(n=4)$. All data are presented as means $\pm S D$, *P $<0.05$, \#P囚0.05. DCFH-DA, 2',7'-Dichlorofluorescin diacetate; ROS, reactive oxygen species; MDA, Malondialdehyde; CAT, Catalase; GPx, Glutathione peroxidase; SA- $\beta$-Gal, senescence associated- $\beta$ galactosidase; TUNEL, terminal deoxynucleotidyl transferase dUTP nick end labeling; DAPI, 4',6diamidino-2-phenylindole. 


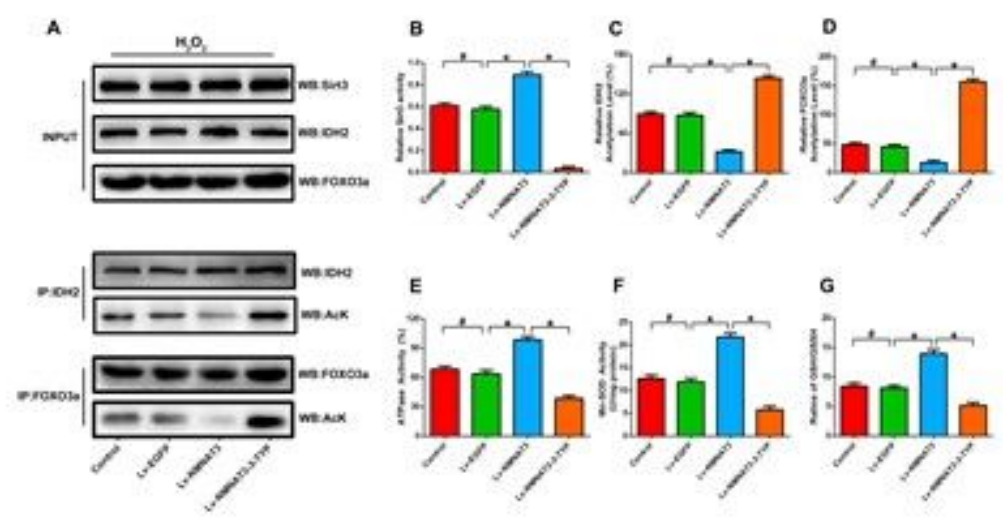

\section{Figure 4}

NMNAT3 enhances the activity of Sirt3 under oxidative stress A,C,D. Western blot analysis of Sirt3, IDH2 and FOXO3a levels in BMSCs. Acetylated IDH2 and FOXO3a were isolated by immunoprecipitation with anti-IDH2 and anti-FOXO3a antibody followed by western blotting with anti-acetyl-lysine antibody $(\mathrm{n}=3)$. B. Detection of Sirt3 relative activity in BMSCs. E. Analysis of ATP synthase activity in BMSCs. F. Analysis of Mn-SOD activity in BMSCs. G. Detection the ratio of GSH/GSSH in BMSCs. All data are presented as means $\pm S D$, $* P<0.05$, \#Pख0.05. Sirt3, silent mating type information regulation 2 homolog $3 ;$ Idh2, isocitrate dehydrogenase 2; FOXO3a, Forkhead-box protein O3a; Mn-SOD, manganese superoxide dismutase; $\mathrm{GSH} / \mathrm{GSSH}$, reduced glutathione/oxidized glutathione. 